\title{
Arzneimittelreaktion: Allergie vs. Intoleranz gegenüber Wirkstoffen
}

In der großen Gruppe der verschiedenen Arzneimittelreaktionen spielen Intoleranzreaktionen eine sehr wichtige Rolle. In der Abgrenzung zu den Arzneimittelallergien, eingeteilt in die Immunreaktionen nach Goombs und Gell, entweder humoral oder zellulär vermittelt, unterscheiden sich Intoleranzreaktionen generell dadurch, dass sie einen anderen pathophysiologischen Hintergrund haben und, anders als bei den Allergien, keine Gedächtniszellen bilden.

Die wichtigsten Gruppen von Intoleranzreaktionen bei Arzneimittelunverträglichkeiten können sowohl die enthaltenen Wirkstoffe als auch die weiteren Bestandteile eines Arzneimittels betreffen. Ein Beispiel für die letztere Gruppe sind Reaktionen auf den Inhaltsstoff Laktose in Arzneimitteln bei hochgradig laktoseintoleranten Personen. In diesem Fall ist das Immunsystem in keiner Weise beteiligt. Weitere Intoleranzreaktionen, die sich nicht gegen die Wirkstoffe richten, können zum Beispiel durch Farbstoffe in Medikamenten ausgelöst werden. Prinzipiell können Intoleranzreaktionen alle Symptome, die auch bei den echten allergischen Arzneimittelreaktionen nach Goombs und Gell in den verschiedenen Gruppen ausgelöst werden, nachahmen. Am häufigsten sind jedoch die urtikariellen Intoleranzreaktionen sowie Intoleranzreaktionen mit der Ausbildung von Rhinitis- oder Asthmasymptomatik. Die häufigste Arzneimittelgruppe, die diese Reaktion auslöst, ist die der nicht-steriodalen Schmerzmittel. Eine Klassifikation zu diesen Hypersensitivitätsreaktionen findet sich im Artikel [1]. Der vermutete Mechanismus ist die Inhibition von COX-1. Der häufigste Auslöser ist ASS. Es besteht eine hohe Kreuzreaktivität gegenüber Diclofenac und Ibuprofen sowie gegenüber anderen NSAID (non-steroidal anti-inflammatory drugs). In einer Untersuchung von GA2LEN in 22 Zentren aus 15 europäischen Ländern betrug die durchschnittliche Prävalenz NSAID-ausgelöster Dyspnoe 1,9\% [2]. Die entsprechende Arzneimittelintoleranz ist daher keineswegs selten. Allerdings fehlen gute Daten zur Häufigkeit der durch NSAID ausgelösten kutanen Beschwerden und der Polyposis nasi. Die Diagnostik der Arzneimittelintoleranzreaktion ist unglücklicherweise nicht einfach, da verlässliche Hauttest und Invitro-Tests fehlen. Es wird zwar empfohlen, eine Prick-Testung durchzuführen, um mögliche IgE-vermittelte Allergien nicht zu übersehen, diese können aber auch die Zusatzstoffe in Medikamenten betreffen. Der Goldstandard bleibt jedoch der doppelblinde placebokontrollierte Provokationstest. Hierzu sind Empfehlungen publiziert [1].

Zusammengefasst: Arzneimittelunverträglichkeitsreaktion im Sinne von Intoleranz sind häufig und sollten regelmäßig in der Differenzialdiagnose mit erwogen werden.
Interessenkonflikt

Der Autor gibt an, dass kein Interessenkonflikt besteht.

Autor

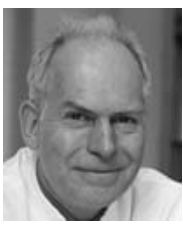

\section{Torsten Zuberbier}

Prof. Dr. med. Dr. h. c., Comprehensive Allergy Center Charité CACC, Klinik für Dermatologie, Venerologie und Allergologie, Charité - Universitätsmedizin Berlin

\section{Korrespondenzadresse}

Prof. Dr. med. Dr. h. c. Torsten Zuberbier Dpt. of Dermatology and Allergy Charité - Universitätsmedizin Berlin Klinik für Dermatologie, Venerologie und Allergologie

Campus Benjamin-Franklin

Allergologie Bereich Prof. Zuberbier

Hindenburgdamm 30/Haus II

12203 Berlin

Deutschland

torsten.zuberbier@charite.de

\section{Literatur}

[1] Kowalski ML, Makowska JS, Blanca M et al. Hypersensitivity to nonsteroidal anti-inflammatory drugs (NSAIDs) - classification, diagnosis and management: review of the EAACI/ENDA(\#) and GA2LEN/HANNA*. Allergy 2011; 66: 818-829. doi:10.1111/ j.1398-9995.2011.02557.x

[2] Makowska JS, Burney P, Jarvis D et al. Respiratory hypersensitivity reactions to NSAIDs in Europe: the global allergy and asthma network (GA2LEN) survey. Allergy 2016; 71 1603-1611. doi:10.1111/all.12941. 